\title{
Exploiting Hub States in Automatic Verification
}

\author{
Giuseppe Della Penna ${ }^{1, \star}$, Igor Melatti ${ }^{1}$, Benedetto Intrigila ${ }^{2}$, \\ and Enrico Tronci ${ }^{3}$
}

1 Dip. di Informatica, Università di L'Aquila, Coppito 67100, L'Aquila, Italy

\{dellapenna, melatti\}@di.univaq.it

2 Dip. di Matematica, Università di Roma "Tor Vergata",

Via della Ricerca Scientifica, 00133 Roma, Italy

intrigil@mat.uniroma2.it

3 Dip. di Informatica, Università di Roma "La Sapienza",

Via Salaria 113, 00198 Roma, Italy

tronci@di.uniroma1.it

\begin{abstract}
In this paper we present a new algorithm to counteract state explosion when using Explicit State Space Exploration to verify protocollike systems.

We sketch the implementation of our algorithm within the Caching Mur $\varphi$ verifier and give experimental results showing its effectiveness.

We show experimentally that, when memory is a scarce resource, our algorithm improves on the time performances of Caching Mur $\varphi$ verification algorithm, saving between $16 \%$ and $68 \%$ (45\% on average) in computation time.
\end{abstract}

\section{Introduction}

State Space Exploration (Reachability Analysis) is at the very heart of all algorithms for automatic verification of concurrent systems.

As well known, the main obstruction to automatic verification of Finite State Systems (FSS) is the huge amount of memory required to complete state space exploration (state explosion).

For protocol and hybrid systems verification, Explicit State Space Exploration often outperforms Symbolic (i.e. OBDD based, 45]) State Space Exploration [1138. Since here we are mainly interested in protocol verification we focus on explicit state space exploration. Tools based on explicit state space exploration are, e.g., SPIN [1723] and Mur $\varphi$ [1119.

In our context, roughly speaking, two kinds of approaches have been studied to counteract (i.e. delay) state explosion: memory saving and auxiliary storage.

In a memory saving approach essentially one tries to reduce the amount of memory needed to represent the set of visited states. Examples of the memory saving approach are, e.g., in [30/7/8/28/26|16 12].

\footnotetext{
^ Corresponding Author: Giuseppe Della Penna. Tel: +390862 433130 Fax: +39 0862
} 433057. 
In an auxiliary storage approach one tries to exploit disk storage as well as distributed processors (network storage) to enlarge the available memory (and CPU). Examples of this approach are, e.g., in [2425].

\subsection{Background}

In 27/10/9 we presented verification algorithms exploiting statistical properties of protocol transition graphs to save on RAM usage as well as to speed up disk based Breadth First (BF) state space exploration. Our algorithms have been implemented within the Mur $\varphi$ verifier. We call $\operatorname{CMur} \varphi$ (Caching Mur $\varphi$ [6]) the resulting verifier.

Shortly, CMur $\varphi$ takes advantage of a statistical property of protocol transition graphs, namely the transition locality. That is, w.r.t the levels of a BF state space exploration, state transitions tend to be between states belonging to close levels of the transition graph. Thus, CMur $\varphi$ replaces the hash table used in a $\mathrm{BF}$ state space exploration with a cache memory (i.e. no collision detection is done) and uses auxiliary (disk) storage for the BF queue. The rationale behind this approach is that a cache maintains only recently visited states. Thanks to transition locality this is sufficient, in most cases, to complete the verification task. Our experimental results [279] show that, with the same amount of RAM, CMur $\varphi$ can verify systems more than $40 \%$ larger than those that can be handled using a hash table based approach. On the other hand, $\operatorname{CMur} \varphi$ verification time can be up to twice that of standard Mur $\varphi$.

Note that CMur $\varphi$ caching techniques is not an alternative to state compression techniques (e.g. hash compaction 28|2616|12]) or to state space reduction techniques (e.g. symmetry and multiset reduction [7]18, partial order reduction [22]). On the contrary, caching is intended to be used together with the available reduction options [279]. The only thing that caching does is storing data in the cache. Such data can be full states, state signatures, or anything else. This is not relevant to the caching schema. This, of course, may be relevant for the effectiveness of the caching schema. As long as the implemented BF search uses a hash table to store visited states (or their signatures) $\operatorname{CMur} \varphi$ caching scheme can be used. For this reason $\operatorname{CMur} \varphi$ can reuse all state reduction procedures implemented in the standard Mur $\varphi$ verifier [27.

\subsection{Goal}

CMur $\varphi$ memory saving stems from the fact the most transitions are local. On the other hand, CMur $\varphi$ time penalty stems from the fact the not all transitions are local. A nonlocal transition leading to a rather old state that has been overwritten (and thus forgotten) in CMur $\varphi$ cache may trigger revisit of large portions of the transition graph and may even lead to nontermination because of loops in the transition graph. The higher $\operatorname{CMur} \varphi$ cache collision rate (i.e. the ratio between collisions and insertions) the higher the probability of revisiting already visited states because of nonlocal transitions.

When the collision rate is high (i.e. close to 1 ) it means that we do not have enough RAM to hold all visited states. So our only hope to decrease the 
time penalty due to revisiting in such a situation is to make a better use of the available RAM.

Quite clearly a (large) fraction of the available RAM must be used to store recently visited states. This is indeed what $\operatorname{CMur} \varphi$ already does. Here we propose to use a (small) fraction of the available RAM to store hub states, that is states that have an in-degree (i.e. number of incoming transitions) much greater than the average in-degree of the set of reachable states. The rationale behind such proposal is that many nonlocal transitions will lead to hub states. Thus avoiding revisiting hub states (and so their successors) may be an effective way to reduce $\mathrm{CMur} \varphi$ time penalty when the collision rate is high.

Note that when the collision rate is low (close to 0 ) it means that we have (almost) enough RAM to store all reachable states. In such a case CMur $\varphi$ does not incur any time penalty. That is, verification with CMur $\varphi$ takes the same amount of time as with standard $\operatorname{Mur} \varphi[19]$.

Unfortunately our goal of storing hub states faces a substantial obstruction: we do not know before hand if a state is a hub or not. Thus, to carry out our goal we need a both time and memory effective way to select hub states among the states visited so far. In other words, the obstruction here is not in storing (the few) hub states, but rather in recognizing that a state seen during the visit is indeed a hub state.

In this paper we show that protocol-like systems do have hub states and present an effective algorithm to select hub states among the states visited so far.

Intuitively, we use a hard to write cache L2, that is a cache in which an insertion request is actually carried out with (a small) insertion probability $p$. This means that states that are frequently seen during our visit will have a greater chance than seldom seen states of actually making their way into L2. As a result, statistically speaking, L2 will tend to store the hub states among the states visited so far. Of course not all hub states will be in L2 nor all states in L2 will be hubs. Still, we can show experimentally that L2 is an effective way to catch hub states.

\subsection{Main Results}

Our main results can be summarized as follows.

In Section 3 we show experimentally that protocol-like systems do have hub states. We support our claim by measuring the distribution of the in-degree of the reachable states for the set of protocols included in the Mur $\varphi$ verifier distribution [19].

In Section 4 we present our algorithm to select hub states among the states visited so far.

In Section 5 we show how an appropriate value for the insertion probability $p$ in L2 can be computed.

We implemented our algorithm within the $\operatorname{CMur} \varphi$ [6] verifier. We call HubCMur $\varphi$ the resulting verifier. In Section [6] we give experimental results on HubCMur $\varphi$ as compared to CMur $\varphi$. Our experimental results show that when 
the collision rate is high typically HubCMur $\varphi$ allows between $16 \%$ and $68 \%$ (45\% on average) of saving in the verification time. Of course when the collision rate is low HubCMur $\varphi$ behaves essentially as $\operatorname{CMur} \varphi$.

\subsection{Related Works}

A rather systematic study of statistical properties of transition graphs is presented in [21]. The author of [21] concludes that there are no hubs in transition graphs. Note however that the definition of hub state used in [21] is different form ours. For us a reachable state $s$ is a hub state if its in-degree is much higher than the average in-degree of the reachable states whereas [21] also requires the $s$ in-degree to be not too smaller than the number of (reachable) states.

Of course what is the right definition of hub depends on the intended application. Anyway, because of this different definition of hub states there is no contradiction between our results about hub existence and those in [21].

Moreover the focus of our paper is not proving or disproving hub existence but rather finding ways to exploit the fact that there are states whose in-degree is much higher that the average one. Finally, the issue of exploiting statistical properties of transition graphs is not investigated in [21].

A survey on caching schemes is presented in [15. Note however that [15] studies Depth First (DF) search with a linked list based hash table. Caching $\operatorname{Mur} \varphi$ 279] instead uses a BF search with an open addressing hash table. As remarked in [15] this is a quite different scenario. In fact, $\operatorname{CMur} \varphi$ caching schema works quite well [279] with open addressing and BF search and does not seem to work with a DF search (SPIN like).

Note that we do not reduce the state space using our hub states. So our approach has nothing to do with Partial Order (PO) reduction [22] techniques. On the other hand we can exploit hub states (if any) in a PO reduced state space.

Finally, [3], using static analysis techniques, studies the issue of which states should be stored in order to save RAM. The results in [3] are orthogonal to ours, note however that the two approaches can be usefully combined.

\section{Background}

In this section we give some basic definitions that will be useful in the following. For our purposes, a protocol is represented as a Finite State System.

\section{Definition 1}

1. A Finite State System (FSS) $\mathcal{S}$ is a 4 -tuple $(S, I, \mathcal{A}, R)$ where: $S$ is a finite set (of states), $I \subseteq S$ is the set of initial states, $\mathcal{A}$ is a finite set (of transition labels) and $R$ is a relation on $S \times A \times S$. $R$ is usually called the transition relation of $\mathcal{S}$.

2. Given states $s, s^{\prime} \in S$ and $a \in A$ we say that there is a transition from $s$ to $s^{\prime}$ labeled with $a$ if and only if $R\left(s, a, s^{\prime}\right)$ holds. The set of successors of state $s$ (notation next $(s)$ ) is the set of states $s^{\prime}$ such that there exists $a \in A$ such that $R\left(s, a, s^{\prime}\right)$ holds. 
3. The set of reachable states of $\mathcal{S}$ (notation $\operatorname{Reach}(\mathcal{S})$ ) is the set of states of $\mathcal{S}$ reachable in 0 (zero) or more steps from $I$. Formally, $\operatorname{Reach}(\mathcal{S})$ is the smallest set such that
1. $I \subseteq \operatorname{Reach}(\mathcal{S})$,
2. for all $s \in \operatorname{Reach}(\mathcal{S}), \operatorname{next}(s) \subseteq \operatorname{Reach}(\mathcal{S})$.

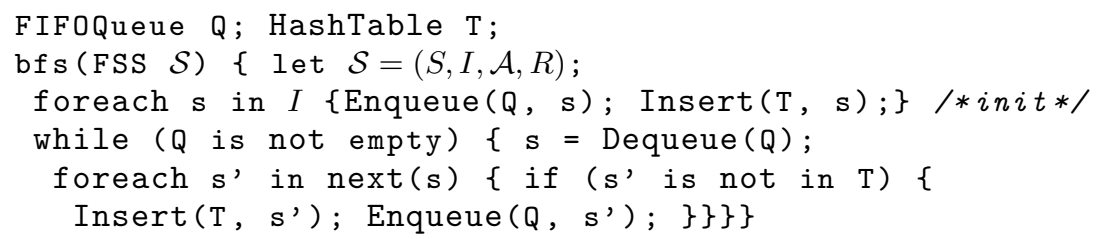

Fig. 1. Basic Breadth First Search

In the following we will always refer to a given system $\mathcal{S}=(S, I, \mathcal{A}, R)$. Thus, for example, we will write $\operatorname{Reach}$ for $\operatorname{Reach}(\mathcal{S})$. Also, we may speak about the set of initial states $I$ as well as about the transition relation $R$ without explicitly mentioning $\mathcal{S}$.

The core of all automatic verification tools is the reachability analysis, that is the computation of Reach given a definition of $\mathcal{S}$ in some language.

Since the transition relation $R$ of a system defines a graph (transition graph), computing Reach means visiting (exploring) the transition graph starting from the initial states in $I$. This can be done, e.g., by using a Depth-First (DF) search or a Breadth-First (BF) search. For example, Mur $\varphi$ [19] and (the latest version of) SPIN 23] may use a DF as well as a BF search.

In the following we will focus on BF search. The Mur $\varphi$ algorithm for the BF visit is shown in Figure 1. Namely, function bf s of Figure1 takes as input a FSS $\mathcal{S}$ and performs a BF visit of $\mathcal{S}$ transition graph. To this end, it uses a FIFO queue $Q$ and a hash table $T$. The first maintains the BF front (i.e. the states to be expanded), while the latter stores the visited states, so avoiding to revisit the same states. Thus, state explosion occurs on T and Q. Finally, note that, if $\mathrm{T}$ and $\mathrm{Q}$ fit in the available memory, bfs will surely terminate, since the set of reachable states is finite.

\section{Hub States}

Inspired by 14229 we call $h u b$ a reachable state which in-degree is much higher than the average in-degree of all reachable states. Note that, as discussed in Section 1.4, our definition of hub state is different from the one used in [21.

In this section we show experimentally that for protocol-like systems hub states do exist. We do this by showing that all our benchmark protocols indeed have hub states. We use as benchmark protocols all those available in the Mur $\varphi$ verifier distribution [19]. The protocols tested cover a wide range of concurrent software typologies such as synchronization, authentication, cache coherence, distributed locks, etc. Thus we have a fairly representative benchmark set. 


\subsection{Measuring Hub States Presence}

In this section we give the basic definitions needed to understand the experimental results in Section 3.2 .

Definition 2. Let $\mathcal{S}=(S, I, A, R)$ be an FSS, and let $s \in S$ be a state. We call in-degree of the state $s$ the number indeg $(s)$ of transitions leading to $s$. That is: $\operatorname{indeg}(s)=|\{(r, a) \in \operatorname{Reach}(\mathcal{S}) \times A \mid R(r, a, s)\}|$.

Our goal is to study the in-degree distribution in protocol-like systems. As usual when reporting statistical results, to make distributions relative to different systems easily comparable we replace the absolute number of states with the fraction $x$ of reachable states and the actual in-degree value with its fraction of the maximum in-degree. In this way all quantities lie in the interval $[0,1]$.

To build the in-degree distribution we proceed in the standard way. Namely, we divide the interval $[0,1]$ in $\left\lceil\frac{1}{\Delta}\right\rceil$ subintervals of length $\Delta$ and, for each subinterval $k$, we compute the fraction of the reachable states whose fraction of the maximum in-degree falls in interval $k$. The following definition gives the formal details.

Definition 3. Let $\mathcal{S}=(S, I, A, R)$ be an FSS, $M_{\text {indeg }}=\max \{\operatorname{indeg}(t) \mid t \in$ $\boldsymbol{\operatorname { R e a c h }}(\mathcal{S})\}$ be the maximum in-degree of $\mathcal{S}$ and $\Delta \in[0,1]$.

- We define function $\theta:(0,1] \times \mathbb{N} \rightarrow[0,1]$ as follows:

$$
\theta(\Delta, k)=\frac{\left|\left\{s \in \boldsymbol{\operatorname { R e a c h }}(\mathcal{S}) \mid(k-1) \Delta M_{\text {indeg }}<\operatorname{indeg}(s) \leq k \Delta M_{\text {indeg }}\right\}\right|}{|\operatorname{Reach}(\mathcal{S})|}
$$

Function $\theta(\Delta, k)$ returns the fraction of reachable states whose in-degree is a fraction $y \in((k-1) \Delta, k \Delta]$ of the maximum in-degree. In other words, $\theta(\Delta, k)$ returns the probability that a reachable state has an in-degree which is a fraction $y \in((k-1) \Delta, k \Delta]$ of the maximum in-degree. Thus, technically speaking, $\theta(\Delta, k)$ is a probability density. Of course, for us, function $\theta(\Delta, k)$ is only interesting when $k \leq \frac{1}{\Delta}$.

- We define function $\tau:(0,1] \times[0,1] \rightarrow[0,1]$ as follows:

$$
\tau(\Delta, x)=\theta\left(\Delta,\left\lceil\frac{x}{\Delta}\right\rceil\right)
$$

We also write $\tau_{\Delta}(x)$ for $\tau(\Delta, x)$ and denote with $\tau_{\Delta}$ function $\lambda x . \tau(\Delta, x)$. That is, $\tau_{\Delta}:(0,1] \rightarrow[0,1]$ is defined as $\tau_{\Delta}(x)=\tau(\Delta, x)$.

Note that function $\tau_{\Delta}$ is completely defined once we know the values $\tau_{\Delta}(\Delta)$, $\tau_{\Delta}(2 \Delta), \ldots \tau_{\Delta}(1)$.

\subsection{Experimental Results About Hub States}

To carry out our plan we modified the $\operatorname{Mur} \varphi$ verifier so as to compute function $\tau$ in Definition 3. Namely, we compute $\tau_{\frac{1}{n}}\left(\frac{1}{n}\right), \ldots, \tau_{\frac{1}{n}}\left(\frac{n-1}{n}\right), \tau_{\frac{1}{n}}(1)$ while performing state space exploration. In our experiments, we set $n=20$. 
Our results are shown in Figure 2 where, for each protocol in our benchmark, we plot $\tau\left(\frac{1}{n}, x\right)$ ( $y$-axes) versus the fraction $x$ of the maximum in-degree ( $x$-axes).

The graphs in Figure 2 show that most reachable states have an in-degree that is a rather small fraction of the maximum in-degree. However there is a small fraction of states that have an in-degree that is close to the maximum in-degree.
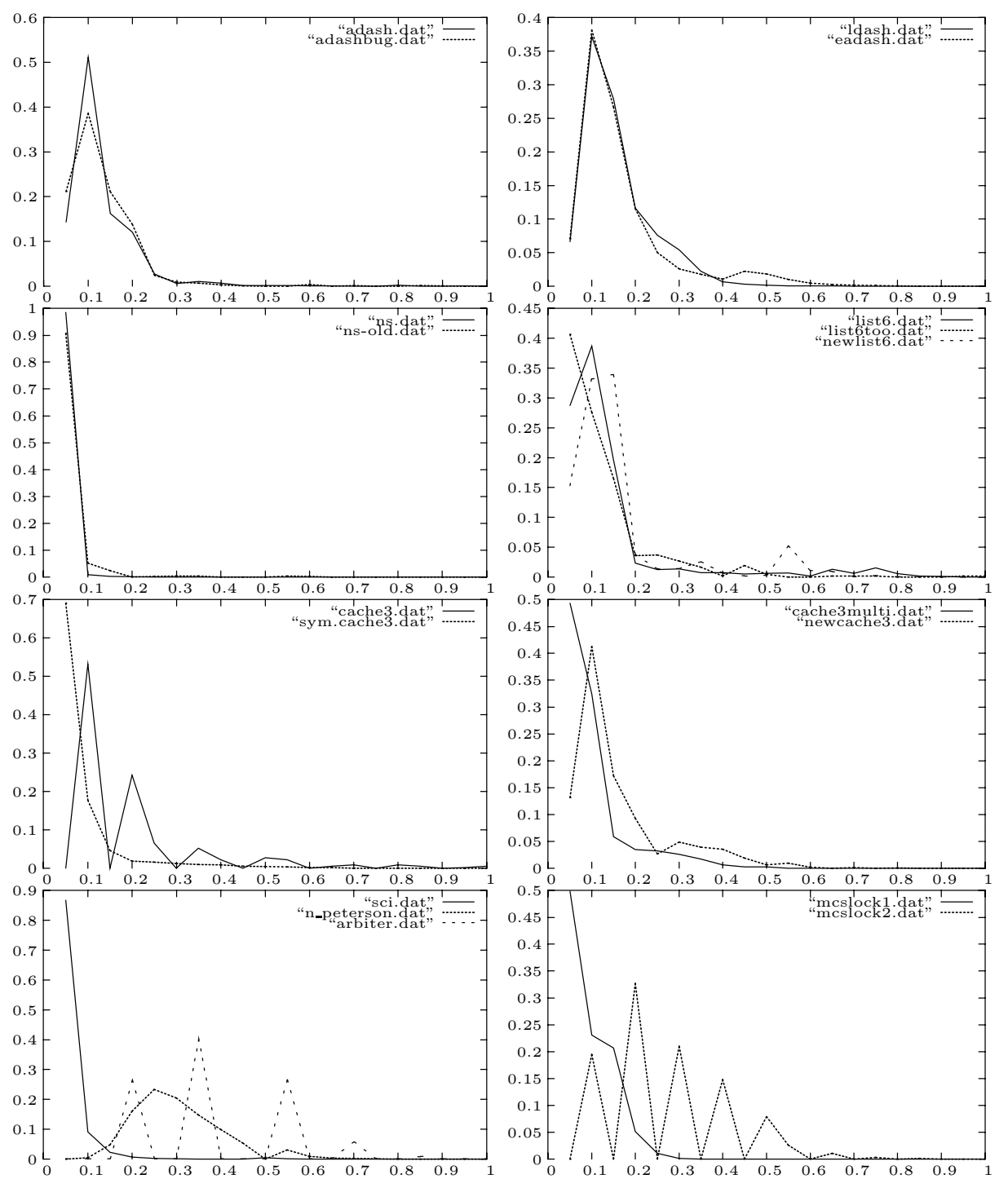

Fig. 2. Density of probability $\tau_{\frac{1}{20}}$ graphs for protocols included in the Mur $\varphi$ distribution. The curves show the fraction of reachable states $y$ which in-degree is a fraction $x$ of the max in-degree. Thus, by definition $y>0$ when $x=1$. Note log scale on $y$ axes. 


\section{Exploiting Hub States in State Space Exploration}

In this section we present an algorithm that is able to effectively select hub states among the states visited so far. Note that the correctness of our algorithm does not depend on the results in Section 3 . However such results will help us to understand why the proposed algorithm is effective on protocol-like systems.

Before describing our algorithm, in Figure 3 we briefly recall the CMur $\varphi$ 9] one. With respect to Figure 1, we have that in Figure 3 the queue is now implemented on disk, and the hash table $\mathrm{T}$ is replaced by a cache. That is, if the insertion of a state $\mathbf{s}^{\prime}$ causes a collision because of a state $t$ already in cache $\mathrm{T}$, then $\mathrm{t}$ is overwritten (and thus forgotten). This implies that, if $\mathrm{t}$ is reached again, it will be revisited, since it is not in our cache anymore. This means that in general cache $\mathrm{T}$ may not be able to prevent nontermination of our visit. As shown in Figure 3, to guarantee termination in $\operatorname{CMur} \varphi$, the main while cycle is guarded by the collision rate, i.e. the ratio between the number of collisions and the number of insertions in cache T. In fact, when the collision rate becomes too high, we are visiting over and over the same set of states. In this case we should give up our verification task because of lack of memory.

In order to improve CMur $\varphi$ time performances, we present a new two-level caching algorithm. The rationale behind this algorithm is the one discussed in Section 1.2

To implement the ideas in Section 1.2 we proceed as follows. We modify the cache based BF algorithm in Figure 3 as shown in Figures 4 and 5 . Namely, we split cache $\mathrm{T}$ in two parts, L1 and L2, with a split ratio $0<p_{h}<1$. Thus, if $M$ was the amount of RAM dedicated to $\mathrm{T}$, then $p_{h} M$ is now dedicated to L1 and $\left(1-p_{h}\right) M$ to L2. In our experiments, we set $p_{h}=0.7$. This is a reasonable value, since hub states are always a very small subset of the reachable states (see Figure 21).

The idea is to use L1 to store the recently visited states, so inheriting the goal of $\mathrm{T}$ (i.e. to exploit transition locality) in $\operatorname{CMur} \varphi$, and L2 (our hard to write cache) to store the hub states. To this end, the algorithm now stores the visited states in L1 (function Insert in Figure 4) and, when the insertion of a state s' causes a collision in L1 on state $t, t$ is passed to L2 before being overwritten. If this causes a collision also in L2 on a state $r, r$ will be overwritten by $t$ with a fixed probability p_ovrwrt (functions Insert $L 2$ and prob_decide in Figure 51). Of course a state is considered visited if it can be found in L1 or L2 (see functions Insert and Lookup L2 in Figures 4 and 5 respectively).

In this way, if state $t$ is a hub, it will have a high probability of being eventually inserted in L2 and remaining there. In fact, since $t$ will be reached more often than the other states, it will be often present and overwritten in L1 and, as a result, it will attempt insertion in L2 many times. This gives $t$ more chances of entering L2 since it will compete more times for the insertion.

We implemented the algorithm of Figures 4 and 5 within the $\operatorname{CMur} \varphi$ verifier [6], calling HubCMur $\varphi$ the resulting verifier. 


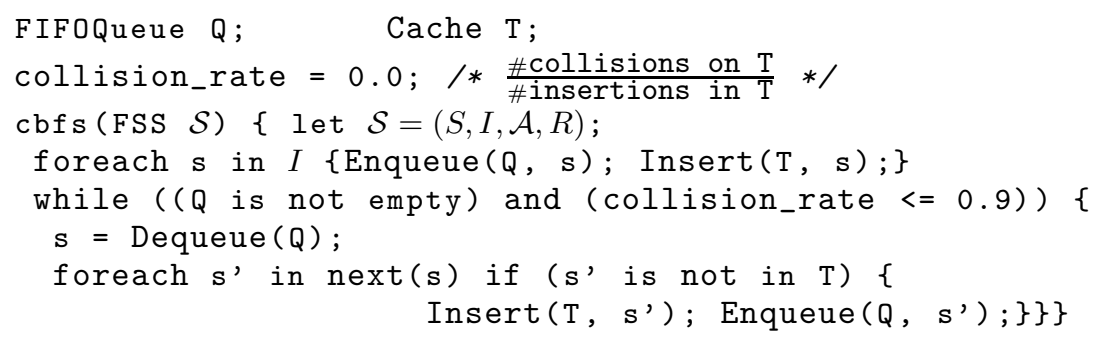

Fig. 3. Cache based Breadth First Search

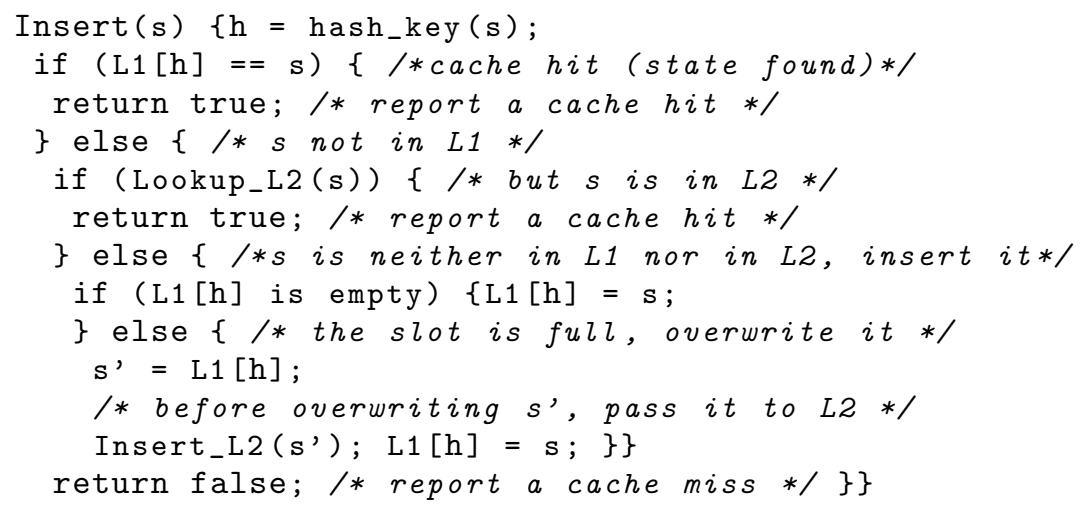

Fig. 4. Function Insert

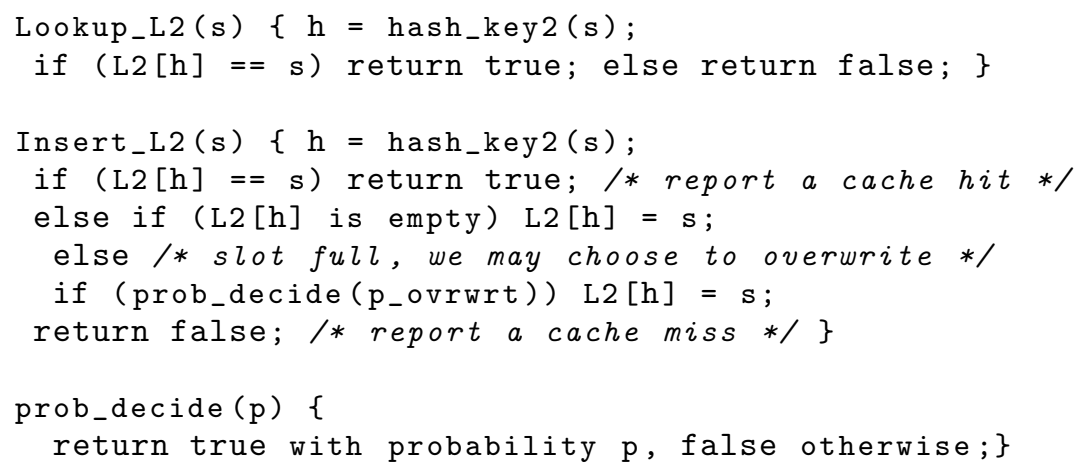

Fig. 5. Functions Lookup_L2, Insert_L2 and prob_decide 


\section{Tuning the Overwrite Probability}

As already said in Section 4, a state that causes a collision in the L2 cache is overwritten with a fixed probability p_ovrwrt. To make L2 effective in finding and retaining hub states, it is important to choose a suitable value of p_ovrwrt.

We carried out a set of experiments to determine a reasonable value for p_ovrwrt. In particular, Figure 6 shows the collision rate as a function of the fraction of visited reachable states for values of p_ovrwrt in $\left\{10^{-4}, 10^{-3}, 10^{-2}, 10^{-1}, 1\right\}$. When p_ovrwrt $\leq 10^{-5}$ the collision rate becomes soon pretty high and the visits stops. This is because when p_ovrwrt s too small it is almost like not having L2 at all. For this reason we only plotted p_ovrwrt in the range $\left\{10^{-4}, 10^{-3}, 10^{-2}, 10^{-1}, 1\right\}$. Note that the protocol set used in these experiments is the same one used in Section [6] to assess performances of our algorithm.

Figure 6 shows that when p_ovrwrt is 1 there are cases in which verification does not terminate. For example this happens for protocols mcslock1, mcslock2 and newlists 6 in Figure 6 .

Note that setting p_ovrwrt to 1 is equivalent two using the standard victim cache approach in processor design [20. However, this does not work in our setting, since in this way the algorithm will overwrite too many states (hubs included) thus leading to nontermination.

On the other hand if p_ovrwrt is too small (namely less than $10^{-4}$ ) then L2 will (almost) never be used and, all in all, we have wasted a fraction $p_{h}$ (see Section 4) of our RAM.

Finally, if p_ovrwrt is small enough, only states that are encountered many times during the exploration process will make their way to L2. Summing up, in our experiments we choose to set p_ovrwrt $=10^{-4}$.

\section{Experimental Results}

We report the experimental results we obtained using HubCMur $\varphi$ (Section 4).

We want to measure how much time and (RAM) memory we can save by using our approach. To make the results from different protocols comparable we proceed as follows.

First, for each protocol we determine the minimum amount of memory needed to complete verification using the $\operatorname{Mur} \varphi$ verifier (namely Mur $\varphi$ version 3.1 from [19).

Let $M$ be the amount of memory and $g$ (in $[0,1])$ be the fraction of $M$ used for the queue (i.e. $g$ is gPercentActive using a Mur $\varphi$ parlance). We say that the pair $(M, g)$ is suitable for protocol $p$ iff the verification of $p$ can be completed with memory $M$ and queue $g M$. For each protocol $p$ we determine the least $M$ s.t. for some $g,(M, g)$ is suitable for $p$. In the following we denote with $M(p)$ such $M$.

Of course $M(p)$ depends on the compression options one uses. Mur $\varphi$ offers bit compression $(-\mathrm{b})$ and hash compaction $(-\mathrm{c})$. However, since in our scenario 

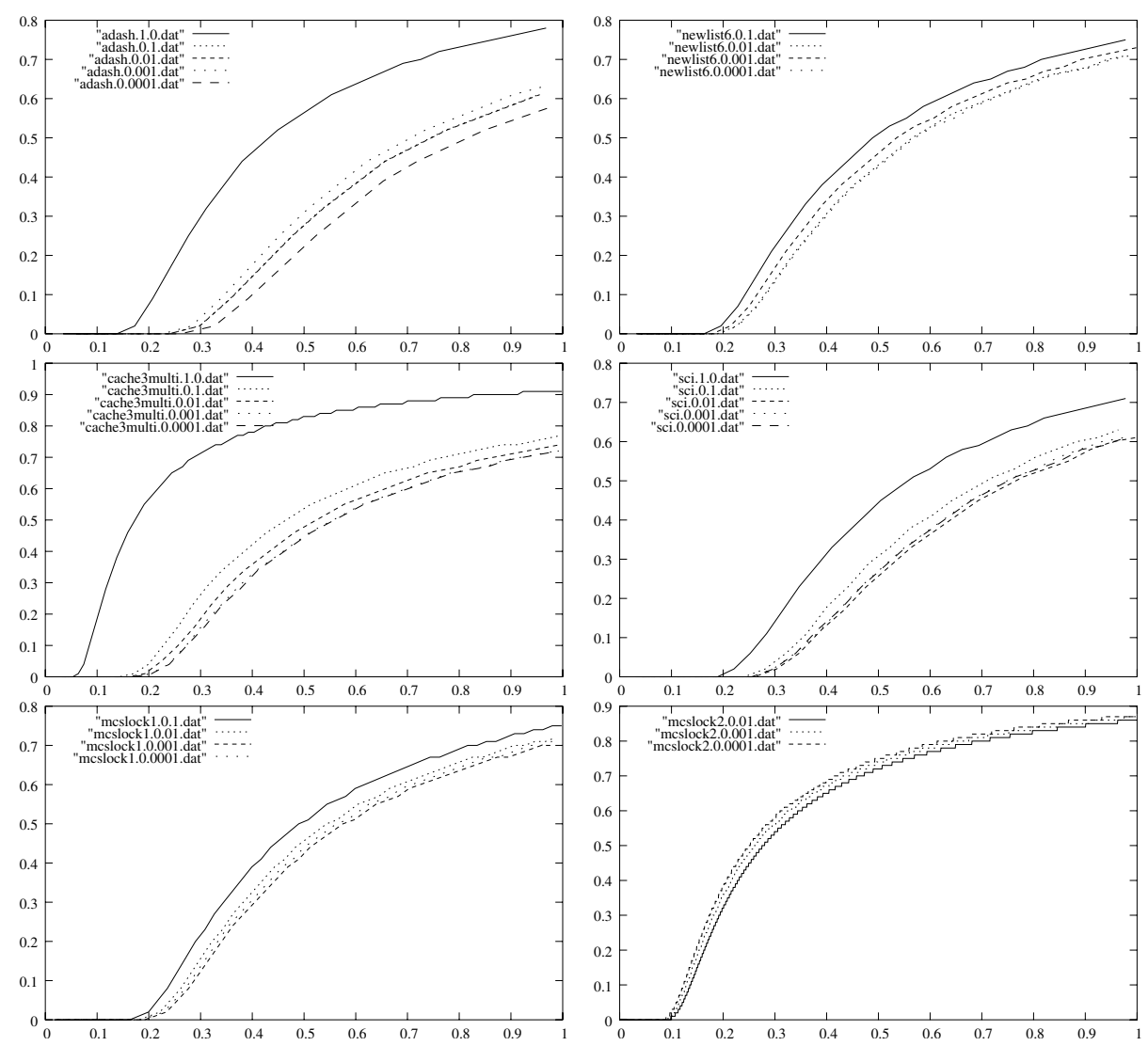

Fig. 6. Collision rate as a function of the fraction of visited protocol states. Each graph shows the collision rate for values of p_ovrwrt in $\left\{10^{-4}, 10^{-3}, 10^{-2}, 10^{-1}, 1\right\}$. A missing line indicates that the verifier was unable to complete the verification with the corresponding value of p_ovrwrt.

RAM is a scarce resource, in the following we only consider the case in which both options are enabled on all verifiers (i.e. $\operatorname{Mur} \varphi, \operatorname{CMur} \varphi, \operatorname{HubCMur} \varphi$ ). Moreover, in order to visit all reachable states, all experiments have been carried out with deadlock detection disabled (-ndl).

Our results are in Figure 7 , where we only show protocols requiring at least 10 kilobytes of RAM and a nonnegligible amount of time to complete state space exploration. In Figure 7, column $\boldsymbol{M}$ gives the minimum amount of memory (in kilobytes) needed to complete state space exploration and column $\boldsymbol{T}$ gives the time (in seconds) to complete state space exploration when using memory $M$. Finally, column Reach gives the number of reachable states.

Our next step is to run each protocol $p$ with less and less memory using both HubCMur $\varphi$ and CMur $\varphi$. That is we run protocol $p$ with memory limits $\alpha M(p), \alpha \in[0,1]$, with the new (L1+L2) and the old (just $\mathrm{CMur} \varphi \mathrm{L} 1)$ cache 
based algorithm. This approach allows us to easily compare the experimental results obtained from different protocols.

The results obtained in such a way are in Fig. 9. Note that in these experiments the value used for $g$ (gPercentActive) is not relevant since the queue is implemented on disk. We give the meaning of rows and columns in Fig. 9.

Column $\alpha$ (with $\alpha \in[0,1]$ ) gives information about the run of protocol $p$ with memory $\alpha M(p)$ (for this reason, the row heading is $\mathbf{M e m}$ ).

Row States gives $\frac{N_{h u b}}{N_{\text {nohub }}}$, where $N_{\text {nohub }}$ is the number of visited states using $\operatorname{CMur} \varphi$ and $N_{h u b}$ is the number of visited states using $\operatorname{HubCMur} \varphi$.

Row Time gives $\frac{T_{h u b}}{T_{n o h u b}}$, where $T_{\text {nohub }}$ is the computation time needed by $\operatorname{CMur} \varphi$ and $T_{h u b}$ is the computation time needed by HubCMur $\varphi$.

A verifier (CMur $\varphi$ or HubCMur $\varphi$ ) is stopped when its collision rate becomes greater than 0.99 . We mark with a $*$ superscript the data obtained when CMur $\varphi$ gives up state space exploration because its collision rate exceeds the given threshold (0.99) and, on the contrary, HubCMur $\varphi$ succeeds in completing the verification. In such cases, instead of giving a ratio, rows States and Time display, respectively, the absolute values for the visited states and the computation time (in seconds) of HubCMur $\varphi$. Note that there was no case in which only CMur $\varphi$ completed the verification.

We are interested in the case in which the collision rate is high, since this means that we do not have enough RAM to store all visited states. For this reason when comparing $\operatorname{CMur} \varphi$ and $\operatorname{HubCMur} \varphi$ performances we only consider the results obtained from the experiments relative to the least $\alpha$ in which both $\operatorname{CMur} \varphi$ and HubCMur $\varphi$ terminate. This means that column $(\alpha-0.01)$ is marked with a $*$ (only HubCMur $\varphi$ terminates). When the collision rate is low (i.e. we do have enough memory to store most of the visited states) CMur $\varphi$ and HubCMur $\varphi$ have similar performances. This can be seen from Figure 9 by looking at the column with the largest value of $\alpha$ (namely the leftmost column).

The experimental results in Figure 9] show that, with respect to CMur $\varphi$, HubCMur $\varphi$ typically saves from $16 \%$ to $68 \%$ (45\% on average) in computation time. Note also that for all protocols there are cases in which, with the available memory, only HubCMur $\varphi$ is able to terminate.

Of course there are protocols (e.g. n_peterson in Figure 9) where HubCMur $\varphi$ is less efficient than CMur $\varphi$. We conjecture that this is due to the shape of the in-degree distribution curves in Figure 2. First, we should note that, technically speaking, the curves in Figure 2 are density of probabilities. Now, for each protocol $p$ we can compare the curve for $p$ in Figure 2 with $\operatorname{HubCMur} \varphi$ performances for $p$ as from Figure 9. From this we see that if the curve of $p$ is rather concentrated (i.e., has a small variance) then HubCMur $\varphi$ performs well on $p$ (e.g., as for protocol sci). On the other hand, if $p$ curve has a large variance (e.g. as for mcslock2 and n_peterson) then $\operatorname{HubCMur} \varphi$ does not perform well on $p$.

We also wanted to test our approach with a large protocol that heavily loads our machine. The results are in Fig. 8. We used protocol sci with parameter MemorySize set to 5. As shown in [10], this protocol has 75,081,011 reachable 


\begin{tabular}{|c|c|c|c|}
\hline Protocol & Reach & M & T \\
\hline n_peterson & 163298 & 813 & 273.32 \\
\hline adash & 10466 & 55 & 62.98 \\
\hline cache3multi & 13738 & 73 & 35.11 \\
\hline newlist6 & 13044 & 67 & 18.34 \\
\hline mcslock1 & 23644 & 120 & 16.76 \\
\hline mcslock2 & 540219 & 2693 & 237.48 \\
\hline sci & 18193 & 94 & 28.17 \\
\hline
\end{tabular}

Fig. 7. Results on a SUN Sparc machine with 512M RAM

\begin{tabular}{|c|c|c|}
\hline Mem & $\mathbf{0 . 4 1}$ & $\mathbf{0 . 3 7}$ \\
\hline States & $80430178^{*}$ & $84045856^{*}$ \\
\hline Time & $47129^{*}$ & $46604^{*}$ \\
\hline \hline Mem & $\mathbf{0 . 3 3}$ & $\mathbf{0 . 2 9}$ \\
\hline States & $92322597^{*}$ & $120543398^{*}$ \\
\hline Time & $51009^{*}$ & $66676^{*}$ \\
\hline
\end{tabular}

Fig. 8. HubCMur $\varphi$ experimental results for protocol sci-31151 with parameter MemorySize $=5$

\begin{tabular}{|c|c|c|c|c|c|c|c|c|c|c|c|c|c|}
\hline \multirow[b]{2}{*}{ mcslock1 } & Mem & 0.60 & 0.59 & 0.58 & 0.57 & 0.56 & 0.55 & 0.54 & 0.53 & 0.52 & 0.51 & 0.50 & 0.49 \\
\hline & States & 0.603 & 0.352 & $72358^{*}$ & $104019^{*}$ & $134834^{*}$ & & & & & & & \\
\hline \multirow{2}{*}{ cache3multi } & Mem & 0.60 & 0.59 & 0.58 & 0.57 & 0.56 & 0.55 & 0.54 & 0.53 & 0.52 & 0.51 & 0.50 & 0.49 \\
\hline & Time & 0.83 & 0.92 & 0.78 & 0.79 & $19.39^{*}$ & $25.22^{*}$ & & & & & & \\
\hline \multirow[b]{2}{*}{ mcslock2 } & Mem & 0.60 & 0.59 & 0.58 & 0.57 & 0.56 & 0.55 & 0.54 & 0.53 & 0.52 & 0.51 & 0.50 & 0.49 \\
\hline & States & 0.99 & 0.976 & 0.956 & 0.93 & 0.919 & 0.885 & 0.805 & 0.714 & $1164348^{*}$ & $1397335^{*}$ & $2085105^{*}$ & \\
\hline \multirow[t]{2}{*}{ newlist 6} & \begin{tabular}{|l|} 
States \\
\end{tabular} & 0.697 & 0.296 & $48882^{*}$ & $63843^{*}$ & & & & & & & & \\
\hline & \begin{tabular}{|l|} 
Time \\
\end{tabular} & 0.75 & 0.32 & $5.14^{*}$ & $6.69^{*}$ & & & & & & & & \\
\hline \multirow{3}{*}{ adash } & \begin{tabular}{|l|} 
Mem \\
\end{tabular} & 0.60 & 0.59 & 0.58 & 0.57 & 0.56 & 0.55 & 0.54 & 0.53 & 0.52 & 0.51 & 0.50 & 0.49 \\
\hline & States & 0.87 & 0.873 & 0.421 & 0.584 & $21362^{*}$ & $32166^{\pi}$ & & & & & & \\
\hline & Time & 0.88 & 0.91 & 0.44 & 0.61 & $11.52^{*}$ & $17.27^{*}$ & & & & & & \\
\hline sci & Mem & 0.49 & 0.48 & 0.47 & 0.46 & 0.45 & 0.44 & 0.43 & 0.42 & 0.41 & 0.40 & 0.39 & 0.38 \\
\hline $\mathrm{n}_{\text {_peterson }}$ & Time & 1.2 & 1.2 & 1.14 & 1.46 & 1.18 & 0.73 & 1.82 & 1.32 & 1.14 & $368.17^{7}$ & & \\
\hline
\end{tabular}

Fig. 9. Comparison of CMur $\varphi$ and HubCMur $\varphi$ experimental results on an INTEL Pentium $3.2 \mathrm{GHz}$ machine with 512M RAM

states and requires 563 Megabytes of memory to be verified with standard Mur $\varphi$ in 35,905 seconds.

CMur $\varphi$ was not able to complete verification with less than 225 Megabytes, that is $40 \%$ of the required (563MB) memory.

On the other hand, as shown in Fig. 8, HubCMur $\varphi$ completed the verification with about $163 \mathrm{MB}$, that is $29 \%$ of the required memory, and a time penalty (w.r.t. standard Mur $\varphi$ with $563 \mathrm{MB}$ of RAM) of $85 \%$.

This suggests that for large protocols $\operatorname{HubCMur} \varphi$ can achieve huge (about $71 \%$ in our example above) memory savings, possibly at the expense of time. This is better than being left with an out of memory message after hours of computation.

\section{Conclusions}

We presented a novel explicit verification algorithm that exploits hub states (Section (3) to save on memory usage (Sections 4, 5). We implemented our algorithm within the $\operatorname{CMur} \varphi$ verifier [6] and call $\operatorname{HubCMur} \varphi$ the resulting verifier.

Our experimental results (Section 6) show that, with respect to $\mathrm{CMur} \varphi$, HubCMur $\varphi$ typically saves from $16 \%$ to $68 \%$ (45\% on average) in computation time. 


\section{Acknowledgments}

We gratefully acknowledge discussing with Alan Hu during CHARME 2001 conference the possibility of using a small victim cache in order to improve CMur $\varphi$ performances. Although in its basic form a victim cache does not meet our goals here, it is also quite clear that our hard to write second level cache is a sort of (lucky) victim cache.

\section{References}

1. A.J. Hu, G. York, and D.L. Dill. New Techniques for Efficient Verification with Implicitly Conjoined BDDs. In 31st ACM/IEEE Design Automation Conference (DAC), San Diego, CA, USA, 1994.

2. Albert-Laszlo Barabasi. Linked. Perseus Publishing, 2002.

3. G. Behrmann, K. G. Larsen, and R. Pelánek. To store or not to store. In Proc. of 15th Int. Conf. on: Computer Aided Verification (CAV), volume 2725 of Lecture Notes in Computer Science, Boulder, CO, USA, July 2033. Springer.

4. R. Bryant. Graph-based algorithms for boolean function manipulation. IEEE Trans. on Computers, C-35(8):677-691, Aug 1986.

5. J. R. Burch, E. M. Clarke, K. L. McMillan, D. L. Dill, and L. J. Hwang. Symbolic model checking: $10^{20}$ states and beyond. Inf. Comput., 98(2):142-170, 1992.

6. Caching murphi web page: http://www.dsi.uniroma1.it/ tronci/cached.murphi.html, 2004.

7. C.N. Ip and D.L. Dill. Better verification through symmetry. In Computer Hardware Description Languages and their Applications, Ottawa, Canada, 1993. Elsevier Science Publishers B.V., Amsterdam, Netherland.

8. G. Della Penna, B. Intrigila, I. Melatti, M. Minichino, E. Ciancamerla, A. Parisse, E. Tronci, and M. Venturini Zilli. Automatic verification of a turbogas control system with the mur $\varphi$ verifier. In Hybrid Systems: Computation and Control, HSCC, Proc., volume 2623 of Lecture Notes in Computer Science. Springer, 2003.

9. G. Della Penna, B. Intrigila, I. Melatti, E. Tronci, and M. Venturini Zilli. Exploiting transition locality in automatic verification of finite state concurrent systems. STTT, 6(4), 2004.

10. G. Della Penna, B. Intrigila, E. Tronci, and M. Venturini Zilli. Exploiting transition locality in the disk based mur $\varphi$ verifier. In Formal Methods in Computer-Aided Design, FMCAD'02, Proc., volume 2517 of Lecture Notes in Computer Science. Springer, 2002.

11. D. L. Dill, A. J. Drexler, A. J. Hu, and C. Han Yang. Protocol verification as a hardware design aid. In Proc. of the 1991 IEEE Int. Conf. on Computer Design on VLSI in Computer \&J Processors. IEEE Computer Society, 1992.

12. Peter C. Dillinger and Panagiotis Manolios. Bloom filters in probabilistic verification. In Formal Methods in Computer-Aided Design, FMCAD, Proc., volume 3312 of Lecture Notes in Computer Science. Springer, Nov 2004.

13. Cindy Eisner and Doron Peled. Comparing symbolic and explicit model checking of a software system. In SPIN Workshop, Proc., Lecture Notes in Computer Science. Springer, 2002.

14. Michalis Faloutsos, Petros Faloutsos, and Christos Faloutsos. On power-law relationships of the internet topology. In SIGCOMM '99: Proc. of the Conf. on Applications, technologies, architectures, and protocols for computer communication, New York, NY, USA, 1999. ACM Press. 
15. Jaco Geldenhuys. State caching reconsidered. In SPIN Workshop, Proc., volume 2989 of Lecture Notes in Computer Science. Springer, 2004.

16. Gerard J. Holzmann. An analysis of bitstate hashing. Form. Methods Syst. Des., 13(3):289-307, 1998.

17. Gerard J. Holzmann. The SPIN Model Checker: Primer and Reference Manual. Addison Wesley Professional, 2004.

18. C. N. Ip and D. L. Dill. Efficient verification of symmetric concurrent systems. In Proc. of the IEEE Int. Conf. on Computer Design: VLSI in Computers and Processors, Cambridge, MA, October 1993. IEEE Computer Society Press.

19. Murphi web page: http://sprout.stanford.edu/dill/murphi.html, 2004.

20. David A. Patterson and John L. Hennessy. Computer architecture: a quantitative approach. Morgan Kaufmann Publishers Inc., 1996.

21. Radek Pelánek. Typical structural properties of state spaces. In SPIN Workshop, Proc., volume 2989 of Lecture Notes in Computer Science. Springer, 2004.

22. D. Peled. Ten years of partial order reduction. In Computer Aided Verification, CAV, Proc., volume 1427 of Lecture Notes in Computer Science. Springer, 1998.

23. Spin web page: http://spinroot.com, 2004.

24. U. Stern and D. Dill. Parallelizing the mur $\varphi$ verifier. In Computer Aided Verification (CAV), Proc, volume 1254 of Lecture Notes in Computer Science. Springer, 1997.

25. U. Stern and D. Dill. Using magnetic disk instead of main memory in the mur $\varphi$ verifier. In Computer Aided Verification (CAV), Proc., volume 1427 of Lecture Notes in Computer Science. Springer, 1998.

26. U. Stern and D. L. Dill. A new scheme for memory-efficient probabilistic verification. In IFIP TC6/WG6.1 Joint Int. Conf. on: Formal Description Techniques for Distributed Systems and Communication Protocols, and Protocol Specification, Testing, and Verification, volume 69 of IFIP Conference Proceedings. Kluwer, 1996.

27. E. Tronci, G. Della Penna, B. Intrigila, and M. Venturini Zilli. Exploiting transition locality in automatic verification. In Correct Hardware Design and Verification Methods, CHARME, Proc., volume 2144 of Lecture Notes in Computer Science. Springer, 2001.

28. U. Stern and D.L. Dill. Improved Probabilistic Verification by Hash Compaction. In Correct Hardware Design and Verification Methods, volume 987, Stanford University, USA, 1995. Springer-Verlag.

29. D. J. Watts. Small Worlds: The Dynamics of Networks Between Order and Randomness. Princeton Univ. Press, Princeton, NJ, USA, 1999.

30. Pierre Wolper and Dennis Leroy. Reliable hashing without collision detection. In Computer Aided Verification (CAV), Proc., volume 697 of Lecture Notes in Computer Science. Springer, 1993. 